\title{
Low-Coherent WDM Reflectometry for Accurate Fiber Length Monitoring
}

\author{
R. Hui, Senior Member, IEEE, J. Thomas, C. Allen, Senior Member, IEEE, B. Fu, and S. Gao
}

\begin{abstract}
A fiber-optic low-coherent reflectometer was developed to accurately monitor fiber length variation. A large lengthcoverage range was obtained by using a fiber Bragg grating array in a wavelength-division-multiplexing configuration. The polarization mismatch-induced signal fading was eliminated by applying a polarization spreading technique at the optical receiver and, therefore, no active polarization adjustment was necessary for long-term measurement.
\end{abstract}

Index Terms-Optical delay lines, optical distance measurement, optical fiber measurement, optical interferometry, optical polarization.

$\mathbf{P}$ RECISE MEASUREMENT of the length of an optical fiber is useful in optic communications as well as in fiber-optic sensors. A popular way to measure fiber length is to use a time-domain reflectometer (OTDR). By evaluating the propagation delay of optical pulses, an OTDR can be used to measure fibers as long as several hundred kilometers. However, the length resolution of a typical OTDR is on the order of meters due to pulsewidth limitations. On the other hand, for high precision measurements, optical low-coherence reflectometers (OLCRs) have been used extensively to measure the distribution of reflections in optical components [1], [2]. The OLCR concept is illustrated in Fig. 1, where a wide-band low-coherent light source is used. Coherent interference happens only when the length difference between the two interferometer arms is shorter than the coherence length of the light source. The length resolution $\Delta z$ of an OLCR is related to the bandwidth $\Delta \lambda$ of the light source by [1]

$$
\Delta z=\frac{1}{2 n} \frac{\lambda^{2}}{\Delta \lambda}
$$

where $\lambda$ is the center wavelength and $n$ is the refractive index. A resolution as fine as $10 \mu \mathrm{m}$ can be achieved [2]. The maximum measurement range of an OLCR is usually limited to a few centimeters, which is mainly determined by the length coverage of the scanning optical delay line. Range extension has been proposed in [3] using a pair of retroreflectors in the optical delay line. By letting the light bounce back and forth between the two retroreflectors for $N$ times, the length coverage range of

\footnotetext{
Manuscript received August 6, 2002; revised September 17, 2002. This work was supported by the National Science Foundation under Grant EPS-9 874732 and by the State of Kansas.

R. Hui, J. Thomas, C. Allen, and B. Fu are with the Department of Electrical Engineering and Computer Science, The University of Kansas, Lawrence, KS 66045 USA.

S. Gao is with the Department of Geology, Kansas State University, Manhattan, KS 66506 USA.

Digital Object Identifier 10.1109/LPT.2002.805842
}

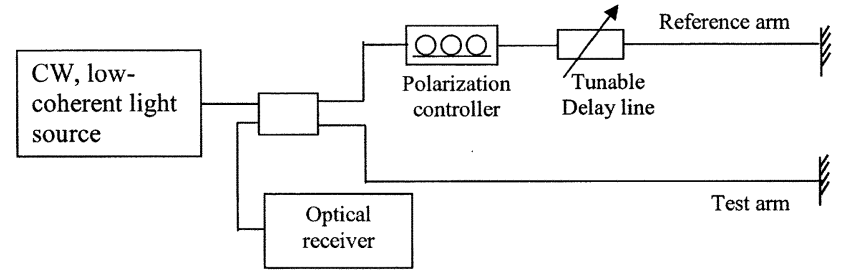

Fig. 1. Basic configuration of an OLCR.

the delay line is increased by $N$ times. In addition to extra insertion loss in the delay line, another disadvantage of this method is that both motor step size and mechanical errors in the translation stage (which controls the position of the retroreflectors in the scanning delay line) will be amplified by $N$ times, degrading the length resolution of the measurement.

Furthermore, since an OLCR is based on coherent interference, it obviously requires the matching of polarization states of the signals reflected from both interferometer arms. Due to the random nature of polarization-mode coupling in an optical fiber, polarization-state mismatch may occur, which causes temporary fading of the coherent beating signal at optical receiver.

A polarization-independent OLCR has been demonstrated [4], where the low coherent light source was first polarized and then launched into an interferometer composed of polarization maintaining (PM) fibers, thus to eliminate the polarization sensitivity. This method may be effective when the fiber arms in the interferometer are relatively short, however for long fiber arms (e.g., a few kilometers), using PM fibers may not be practical.

In this letter, we propose and demonstrate an alternative method to extend the measurement length coverage of an OLCR using a fiber Bragg grating (FBG) array and wavelength-division multiplexing (WDM). Meanwhile, we also introduce a polarization spreading technique in the optical receiver to eliminate the polarization mismatch-induced signal fading. This makes it possible for an OLCR to be used in long-term monitoring of fiber length variations, which has a wide range of applications in fiber-optic sensors such as the monitoring of the earth movement.

The configuration of the proposed OLCR is shown in Fig. 2. Several broad-band continuous-wave light-emitting diodes (LEDs), each with a different central wavelength, can be combined through a WDM multiplexer. A number of FBGs are used and connected in tandem, each reflecting at a different wavelength. A scanning optical-delay line with the maximum tuning range $l_{t}$ is used to vary the length of the reference arm. A tunable optical filter is used at the receiver side for wavelength selection. At a certain wavelength, the length of the reference 


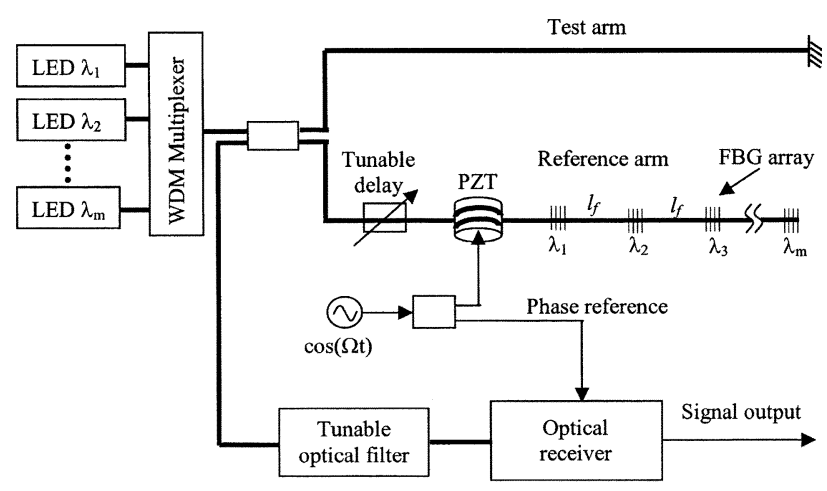

(a)

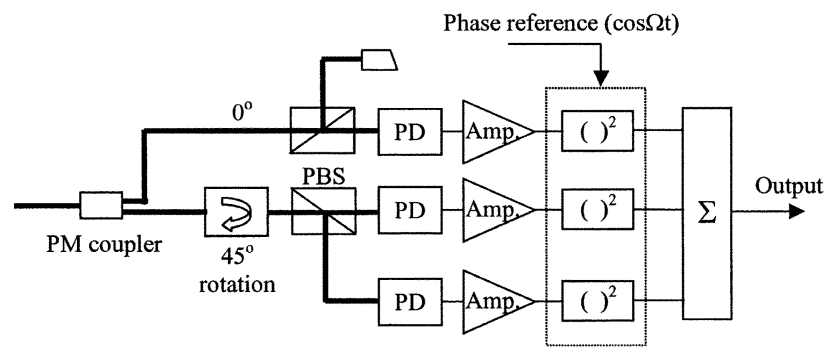

(b)

Fig. 2. Proposed OLCR to extend length coverage and eliminate polarization sensitivity. (a) OLCR configuration using WDM and FBG array. (b) Optical-receiver design with polarization spreading.

arm is determined by the location of the FBG reflecting at that particular wavelength. In this configuration, although the maximum tuning range $l_{t}$ of the optical delay line may be limited by physical constrains, the overall measurable length coverage is approximately $L=M l_{f}$, where $M$ is the total number of FBGs. As a practical concern, in order to ensure a continuous length coverage, the distance between adjacent FBGs $l_{f}$ should be slightly shorter than $l_{t}$. The length resolution of this OLCR is determined by the bandwidths of the optical filter and fiber gratings. According to (1), in the 1550 -nm wavelength band, the combined optical bandwidth of the tunable optical filter and each FBG has to be larger than $0.8 \mathrm{~nm}$ in order to achieve a submillimeter length resolution.

The implementation of polarization spreading technique in the optical receiver is illustrated in Fig. 2(b). In this receiver, the combined optical signal reflected from the two interferometer arms is first split by a 3-dB polarization-maintaining beam splitter. One of the beam-splitter outputs is connected to a linear horizontal polarizer and then detected by a photodiode. Signal from the other output port of the beam splitter is connected to a polarization beam splitter (PBS) via a $45^{\circ}$ polarization rotation patch cord and then the two outputs from the PBS are each detected by a photodiode. After amplification and envelope detection, low frequency electrical signals from the three photodiode branches are added together to form the output.

Using a Monte Carlo simulation, a statistical distribution of receiver photo current can be found as shown in Fig. 3(a), which displays 5000 simulated samples. The probability distribution is also shown in the same figure. Fig. 3(a) demonstrates a high probability of severe signal fading with direct detection using single photodiode. Indeed, this is a well known problem for coherent optical receivers and a detailed analysis can be found in [1].

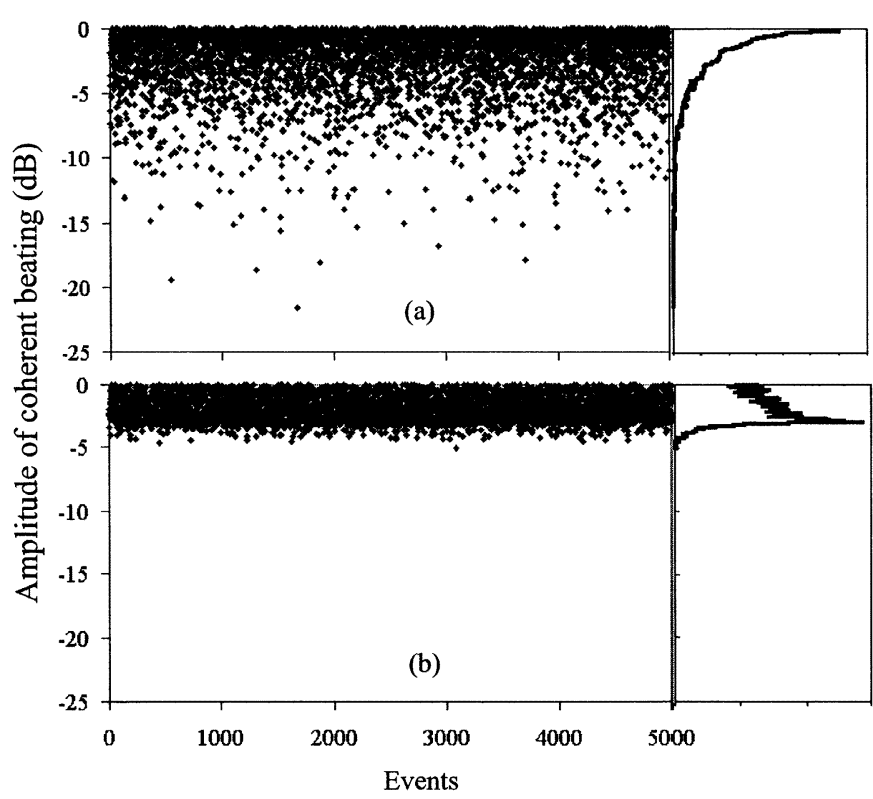

Fig. 3. Monte Carlo simulation of signal fading induced by polarization state mismatch. (a) Using a conventional receiver with a single photodiode. (b) Using the proposed polarization spreading in the optical receiver. The continuous curve at the right-hand side of each plot represents the corresponding probability distribution.

It is not possible for these three photocurrent components to fade simultaneously. Fig. 3(b) shows 5000 simulated samples of the statistical distribution of the total photocurrent $I=$ $I_{1}+I_{2}+I_{3}$. In this case, the maximum variation of the total photocurrent $\mathrm{I}$ is approximately $5 \mathrm{~dB}$. For many applications in the measurement of long-term fiber length variations, a polarization spreading optical receiver can simplify the measurement procedure by eliminating the necessity of endless polarization adjustments.

In order to prove the concept, an experiment was performed using four FBGs with peak reflection wavelengths at 1550 , 1553,1556 , and $1559 \mathrm{~nm}$, respectively. The optical bandwidth of each FBG is approximately $1.2 \mathrm{~nm}$ and the step motor-controlled tunable optical filter at the receiver has an optical bandwidth of $1 \mathrm{~nm}$. The four FBGs were spliced together in tandem. To precisely measure the spacing between adjacent FBGs, we injected a short optical pulse (100 ps) into this FBG string. By tuning the wavelength of the optical pulse, the reflection delay from each FBG can be measured as shown in Fig. 4, which indicates the FBG spacings of 94,75 , and $72 \mathrm{~mm}$. The in-band reflection loss of each FBG is typically $0.2 \mathrm{~dB}$. The pulse amplitude decreases for approximately $0.8 \mathrm{~dB}$ after each FBG, which is mainly caused by splicing between FBGs. This loss can be further reduced if writing FBGs on a single fiber. In addition, this FBG delay line does not require precise mechanical alignment as compared to the method used in [3]. The step-motor-driven scanning optical delay line used in the experiment has the maximum tuning range of $160 \mathrm{~mm}$ in the air. Considering the refractive index difference between air and fiber $(n=1.5)$, this tuning range is equivalent to approximately $106 \mathrm{~mm}$ in the fiber. The FBG array extended the total measurement length coverage to $347 \mathrm{~mm}$. In principle, longer length coverage can be achieved by using a larger 


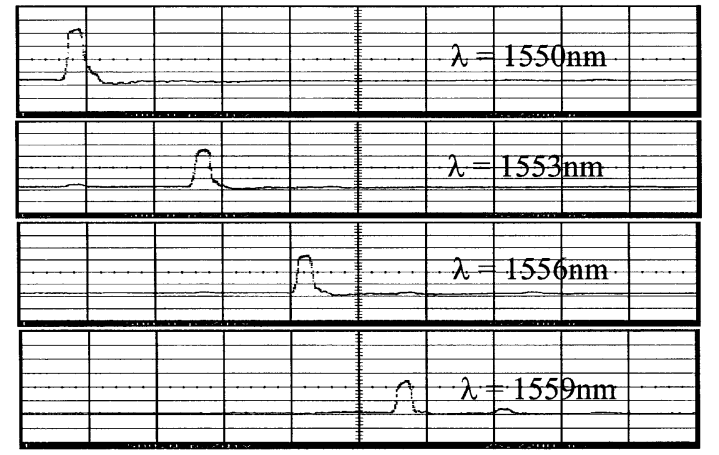

Fig. 4. Measured propagation delay of an optical pulse reflected from FBG string at each different wavelength. Vertical axis: linear scale with arbitrary unit. Horizontal axis: 500 ps/div. Input pulsewidth: 100 ps.

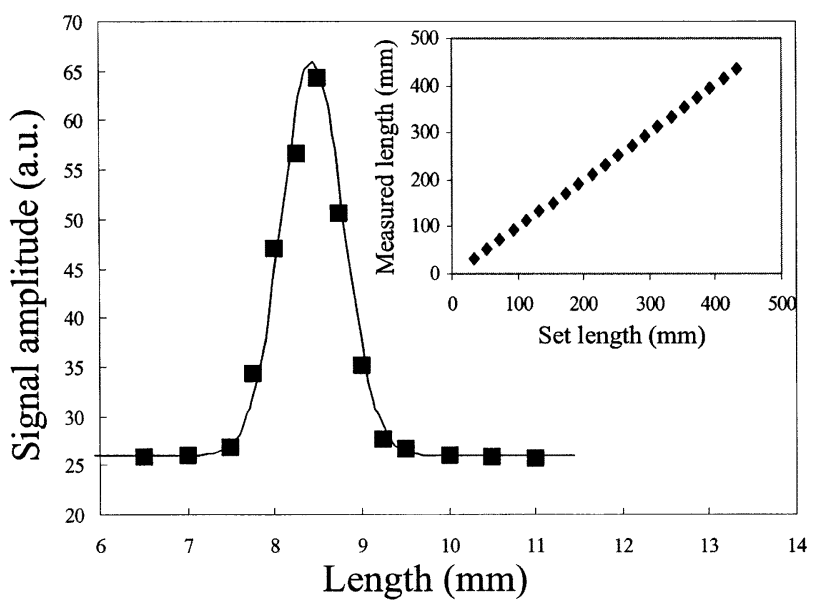

Fig. 5. Measured envelope of the coherent interference using the setup shown in Fig. 2. Inset: Comparison between the measured and the set optical length in the entire length-coverage range.

number of FBGs. For the low-coherent light source, we used a conventional erbium-doped optical fiber amplifier (EDFA) without an input optical signal. The wide-band amplified spontaneous emission (ASE) noise provided by this EDFA covers the wavelength range from 1532 to $1560 \mathrm{~nm}$, which is sufficient for this experiment. The effective optical bandwidth of the cascaded FBG and tunable optical filter is approximately $1 \mathrm{~nm}$, which is much smaller than the total bandwidth of the optical source. The spatial resolution of the measurement can then be estimated to be $0.8 \mathrm{~mm}$ using (1). Fig. 5 shows the measured envelope of the coherent interference using the setup shown in Fig. 2, indeed the spatial linewidth of this measured interference pattern (full-width at half-maximum) is approximately $0.8 \mathrm{~mm}$. If a higher spatial resolution is required, the optical bandwidths of FBGs and the optical filter will have to be increased. This may require a wider bandwidth of the optical source, which can be achieved by wavelength multiplexing as shown in Fig. 1. The inset in Fig. 5 shows the comparison between the actual and the measured length in the whole-length-coverage range. In the measurement, the actual length was varied by manually adjusting an optical delay line.

Fig. 6 shows the results of continuous fiber length monitoring for about three days. In this measurement, each of the two

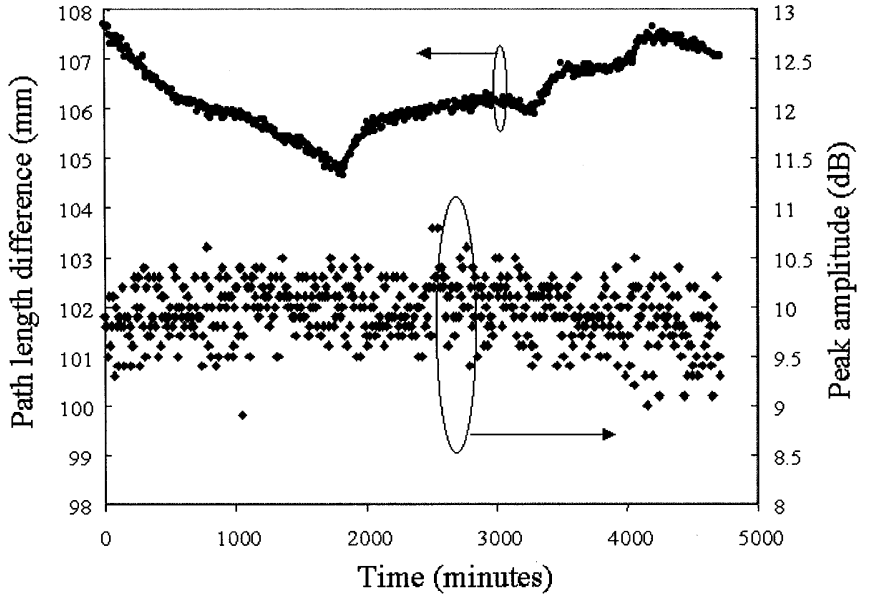

Fig. 6. Results of continuous fiber length monitoring for 5000 minutes. Dots: Measured fiber length versus time. Diamonds: Coherent interference peak amplitude versus time.

interferometer arms is made by a $1-\mathrm{km}$ large effective area fiber (LEAF) cable with a dispersion of $2.5 \mathrm{ps} / \mathrm{nm} \cdot \mathrm{km}$ in the 1550-nm-wavelength window. Fig. 6 shows that the relative length change between the two fiber arms is approximately $3 \mathrm{~mm}$ during the $5000 \mathrm{~min}$ of measurement. We believe this length variation is caused by the relative temperature difference between the two fiber spools. Fig. 6 also shows the measured coherent interference peak amplitude versus time. Although there is no polarization controller used in the setup, signal fading never occurred during the three days although the interference peak amplitude varied by about $3 \mathrm{~dB}$. This variation is somewhat smaller than that theoretically predicted as shown in Fig. 3(b). This may be caused by the depolarization in the optical fiber because of the wide optical signal bandwidth.

In conclusion, we have proposed and demonstrated a fiber-optic OLCR to accurately monitor fiber length variations. In this configuration, an FBG array converted spectral-domain information into spatial-domain and, therefore, extended the total length-coverage range. The polarization mismatch-induced signal fading was eliminated by introducing a polarization spreading technique at the optical receiver. No active polarization control was required. Because of the bandwidth limitation induced by optical filtering, this demonstrated setup is most suitable for applications which require submillimeter spatial resolutions.

\section{REFERENCES}

[1] D. Derickson, Ed., Fiber Optic Test and Measurement. Englewood Cliffs, NJ: Prentice-Hall, 1998, ch. 10.

[2] Y. Gottesman, E. Rao, H. Sillard, and J. Jacquet, "Modeling of optical low coherence reflectometry recorded Bragg reflectograms: Evidence to a decisive role of Bragg spectral selectivity," J. Lightwave Technol., vol. 20, pp. 489-493, Mar. 2002.

[3] K. Takada, H. Yamada, Y. Hibino, and S. Mitachi, "Range extension in optical low coherence reflectometry achieved by using a pair of retroreflectors," IEE Electron. Lett., vol. 31, no. 18, pp. 1565-1567, 1995.

[4] M. Kobayashi, H. Hanafusa, K. Takada, and J. Noda, "Polarization-independent interferometric optical-time-domain reflectometer," J. Lightwave Technol., vol. 9, pp. 623-628, May 1991. 